\title{
Le sujet-lecteur face aux Liaisons dangereuses à l'âge des réseaux sociaux : stratégies d'actualisation et de contextualisation
}

How Can the Reader Comprehend Laclos's Les Liaisons dangereuses at the Age

of Social Networks? Strategies of Actualization and Contextualization

Dominique Hölzle

\section{(2) OpenEdition}

Journals

Édition électronique

URL : http://journals.openedition.org/recherchestravaux/952

ISSN : 1969-6434

Éditeur

UGA Éditions/Université Grenoble Alpes

Édition imprimée

ISBN : 978-2-37747-014-3

ISSN : 0151-1874

Référence électronique

Dominique Hölzle, «Le sujet-lecteur face aux Liaisons dangereuses à l'âge des réseaux sociaux :

stratégies d'actualisation et de contextualisation », Recherches \& Travaux [En ligne], 91 | 2017, mis en ligne le 01 octobre 2017, consulté le 08 septembre 2020. URL : http://journals.openedition.org/ recherchestravaux/952

Ce document a été généré automatiquement le 8 septembre 2020

(C) Recherches \& Travaux 


\title{
Le sujet-lecteur face aux Liaisons dangereuses à l'âge des réseaux sociaux : stratégies d'actualisation et de contextualisation
}

\author{
How Can the Reader Comprehend Laclos's Les Liaisons dangereuses at the Age \\ of Social Networks? Strategies of Actualization and Contextualization
}

Dominique Hölzle

1 Nous nous proposons de réfléchir aux rapports entre actualisation ${ }^{1}$ et contextualisation à propos de l'étude en classe de première des Liaisons dangereuses (1782) de Choderlos de Laclos. Il s'agira de montrer que l'actualisation permet des formes de contextualisation efficace du texte littéraire, dans un double mouvement de rapprochement du texte avec l'expérience subjective de l'élève, pour qu'il en saisisse les enjeux sur un plan à la fois affectif et intellectuel, puis de distanciation de manière à resituer le texte face aux problématiques de l'époque de sa création. Nous développerons tout d'abord une réflexion conceptuelle sur les diverses modalités d'actualisation en classe, en distinguant l'actualisation contextualisante, l'actualisation modernisante et la contextualisation historique. Puis nous présenterons certaines propositions d'étude possibles dans le cadre d'une approche subjective et actualisante du roman de Laclos².

\section{Actualisation contextualisante et actualisation modernisante des œuvres}

2 L'enjeu de l'actualisation dans le cadre de la classe consiste à rechercher ce qui, dans l'horizon culturel actuel des élèves, leur permet d'appréhender, non pas de manière intellectuelle et distanciée, mais sur un plan esthétique, affectif et éthique, les enjeux du texte. Le travail de l'enseignant implique qu'il identifie ce qui, dans le texte, est 
profondément opaque pour un lecteur contemporain, pour chercher ensuite des biais d'actualisation. Il s'agira notamment de rapprocher l'œuvre des représentations et de l'imaginaire des élèves, soit en utilisant un média «moderne » pour leur permettre de s'approprier le contexte historique - nous parlerons alors d'actualisation contextualisante transmédiatique -, soit en montrant la pertinence de cette œuvre au regard de problématiques contemporaines - nous parlerons alors d'actualisation modernisante.

\subsection{L'actualisation contextualisante transmédiatique}

3 La première stratégie d'actualisation consiste à utiliser un média moderne pour reconstituer la réalité de l'époque et favoriser le travail de projection des élèves dans l'imaginaire du texte. L'actualisation contextualisante transmédiatique reste fidèle au contexte historique de l'œuvre, mais repose sur une actualisation des moyens de la représentation.

\subsubsection{L'actualisation contextualisante transmédiatique illustrative}

4 La méthode la plus fréquente consiste à s'appuyer sur des adaptations récentes des œuvres, par le biais d'un média «moderne»: on peut alors parler d'actualisation contextualisante transmédiatique illustrative. Pour Les Liaisons dangereuses, on peut penser aux films de Stephan Frears (Les Liaisons dangereuses, 1988) ou de Milos Forman (Valmont, 1989). Nombreuses sont les adaptations cinématographiques d'œuvres patrimoniales: les adaptations de Molière par Roger Coggio, L'Avare (1980) de Jean Girault, Le Colonel Chabert (1994) d'Yves Angelo, Emma Bovary (1991) de Claude Chabrol, Le Parfum (2006) de Tom Tykwer, le Cyrano (1990) de Jean-Paul Rappeneau, etc. Les bandes dessinées sont aussi un intéressant support pour les actualisations contextualisantes ${ }^{3}$. Ce type d'actualisation repose sur l'idée qu'une grammaire visuelle moderne efface en partie la distance qui sépare l'œuvre du lecteur et permet de reconstituer le cadre historique du texte, ce qui devrait entraîner une adhésion plus directe à l'univers de la fiction. Cependant, les adaptations restent souvent des hommages figés à la grandeur patrimoniale de l'œuvre, qui peut paradoxalement en devenir plus intimidante encore, d'où une accentuation possible du fossé entre l'œuvre et l'expérience subjective de l'élève. Par ailleurs, une telle démarche entraîne forcément un appauvrissement du rapport subjectif au texte, avec l'imposition d'un imaginaire et d'une interprétation particulière. Ces écueils sont moins manifestes avec une deuxième stratégie transmédiatique, que l'on pourrait appeler l'actualisation contextualisante immersive.

\subsubsection{L'actualisation contextualisante transmédiatique immersive}

5 Il s'agit là de faire visionner tout ou partie d'une œuvre autonome, qui illustre cependant le contexte historique ou culturel de l'œuvre. On peut penser au Barry Lyndon (1975) de Stanley Kubrick, à Ridicule (1996) de Patrice Leconte pour les œuvres du xvIII siècle, ou à Excalibur (1981) de John Boorman pour le contexte du roman arthurien. Le parasitage de l'imaginaire de l'élève par les représentations imposées de l'œuvre, s'il n'est pas absent, est nécessairement moins important que dans le cas de l'actualisation contextualisante illustrative; se trouve aussi évité l'effet d'intimidation 
que peut avoir la stèle cinématographique érigée à la gloire de l'œuvre patrimoniale. À côté de cette reconstitution du passé par des moyens modernes, l'approche actualisante peut aussi consister à trouver dans notre époque des situations qui éclairent les intrigues, les dilemmes et les problématiques de l'œuvre du passé. Dans une perspective plus proche des concepts développés par Yves Citton, il ne s'agit plus de rendre vivant le passé en l'illustrant, il s'agit de montrer que le contexte contemporain permet de comprendre le contexte de l'époque du texte, et inversement. On peut alors parler d'actualisation modernisante.

\subsection{L'actualisation modernisante}

6 Là encore, il existe deux approches : l'actualisation modernisante objective, centrée sur l'objet étudié, et celle qui s'inscrit dans un horizon culturel plus vaste, et même sur les expériences vécues des élèves, qu'on pourrait nommer actualisation modernisante subjective. Précisons que si l'actualisation modernisante est fréquemment transmédiatique, elle ne l'est pas forcément, puisque l'enseignant peut notamment s'appuyer sur l'essor du genre romanesque "Young Adults» (comme la série romanesque des Twilight, des Hunger Games, ou encore des Divergente) pour montrer la pertinence de problématiques abordées par des textes anciens qui se retrouvent dans ces romans populaires ${ }^{4}$.

\subsubsection{L'actualisation modernisante objective}

7 Pour montrer que l'œuvre reste vivante, et peut s'actualiser, les Liaisons offrent de nombreuses possibilités: l'œuvre a été de nombreuses fois modernisée, sous forme romanesque, avec Nous sommes cruels de Camille de Peretti (2006), mais aussi, de manière transmédiatique, sous forme théâtrale avec Quartet (1980) de Heiner Müller, et au cinéma, avec le film de 1960 de Roger Vadim ${ }^{5}$. C'est surtout Cruel Intentions (1999) de Roger Kimble, qui nous semble exploitable en classe dans la perspective d'une actualisation modernisante objective. Ce film américain, dont la version française est subtilement intitulée Sexe Intentions, transpose en effet habilement le récit de Laclos dans le milieu de la jeunesse dorée du New York contemporain ${ }^{6}$. Le problème peut cependant provenir d'une forme d'obsolescence rapide des images ainsi modernisées, au point que l'œuvre pourrait paraitre encore plus distante pour les jeunes spectateurs qu'elle ne le serait avec une stratégie d'actualisation contextualisante illustrative ${ }^{7}$. Une autre stratégie consiste à trouver des œuvres différentes, ou des phénomènes culturels contemporains, qui reposent sur des problématiques comparables à celles de l'œuvre originale, et qui permettent d'actualiser les enjeux, les dilemmes, les conflits du texte source.

\subsubsection{L'actualisation modernisante subjective}

Dans cette perspective, il s'agira de trouver des œuvres modernes susceptibles de permettre aux élèves de saisir les enjeux et les problématiques de textes anciens, tout en comblant le fossé qui les sépare habituellement des œuvres patrimoniales. Dans ce travail de désacralisation, le Marie-Antoinette (2006) de Sofia Coppola constitue un objet hybride qui peut être utile pour permettre aux élèves de s'approprier le contexte des Liaisons dangereuses: luxueux film en costume, il pourrait être associé aux œuvres que 
nous avons précédemment désignées comme des exemples d'actualisation contextualisante immersive, mais les nombreux anachronismes ${ }^{8}$ voulus par la cinéaste contribuent à rapprocher l'œuvre des référentiels de l'élève. Concernant Les Liaisons dangereuses, le roman peut être rapproché avec profit des intrigues et des personnages de la série télévisée Gossip Girls, qui se déroule dans le monde privilégié des private schools new-yorkaises: séductions, manipulations, vengeances, messages anonymes, arrogance de classe sont autant d'éléments qui permettent d'établir des ponts entre les deux œuvres?.

9 Mais dans cette démarche d'actualisation, il n'est pas forcément nécessaire d'utiliser une œuvre précise. On peut également s'appuyer sur les rituels médiatiques et sur la vie sociale des adolescents. Si l'on s'intéresse au vécu des élèves, comment leur faire percevoir ce clivage fondamental entre le moi privé et l'être social, la constante négociation entre les deux, qui constitue l'essentiel de la thématique de la tradition du roman d'analyse français, qui remonte au moins à La Princesse de Clèves, et qui, de fait, joue un rôle capital dans Les Liaisons dangereuses? Il nous semble que les réseaux sociaux constituent un biais d'actualisation intéressant, biais d'autant plus légitime que, comme le montre ici-même Maria Léone, les problématiques liées au développement des réseaux sociaux avaient déjà été pensées de manière plus ou moins directe par Diderot, Richardson et Rousseau. La conscience d'être constamment en représentation, on la trouve dans les lettres des libertins comme sur les messages du Mur de Facebook; quant à la question de la popularité dans les cercles lycéens, on peut la lier à la problématique de la gloire du libertin ${ }^{10}$; et on peut aussi s'appuyer sur la différence entre les messages privés et les messages du Mur pour séparer les correspondances publiques des correspondances secrètes dans l'œuvre de Laclos. La cruauté méprisante de $\mathrm{M}^{\mathrm{me}}$ de Merteuil à l'égard de ses victimes moquées (notamment $\mathrm{M}^{\mathrm{me}}$ de Tourvel et Cécile) peut également amener à une réflexion sur la question du harcèlement, qui sera d'autant plus efficace que le détour aura été pris en empruntant les chemins de la fiction. On peut ainsi amener les élèves à s'interroger sur les contraintes du moi social, sur les enjeux et les dangers de l'image projetée, dans les réseaux sociaux en particulier.

Évidemment, l'actualisation n'est pas sans poser de sérieux problèmes d'aplatissement, d'appauvrissement ou d'affadissement. La difficulté est pour l'enseignant de réussir à faire percevoir la singularité, la puissance de sidération d'un texte, à son époque mais aussi à la nôtre. Au terme de ce premier mouvement de rapprochement de l'œuvre de l'univers culturel des élèves, on peut espérer que le texte aura été désacralisé, qu'il apparaîtra désormais comme un corps vivant, appropriable et manipulable, avec lequel on pourra interagir. Peut alors commencer un nouveau mouvement, plus distancié, un mouvement de contextualisation historique, afin de faire revivre le texte dans son époque.

\subsection{La contextualisation historique}

11 Nous sommes là dans un domaine familier pour les enseignants de lettres. On pourra s'appuyer sur les lectures cursives et les groupements de textes, pour présenter des œuvres qui éclairent les enjeux éthiques et esthétiques des Liaisons (Les Égarements ou les dialogues de Crébillon, le théâtre de Marivaux, des extraits des œuvres des moralistes, de romans libertins mondains, de la Nouvelle Héloïse de Rousseau ou des romans de Madame Riccoboni pour comprendre l'importance du sentiment). Sont 
également possibles des recherches thématiques au CDI sur la sociabilité, sur les rapports entre le libertinage mondain et le libertinage érudit, des recherches iconographiques, sur Watteau, Fragonard, Boucher, sur le rococo, etc. Une fois ce travail de contextualisation achevé, on peut passer aux stratégies pédagogiques pour encourager la lecture subjective, et l'actualisation des problématiques, dans la perspective décrite par Yves Citton dans Lire, interpréter, actualiser.

\section{Propositions de lectures subjectives et actualisantes des Liaisons dangereuses}

Il s'agit ici de voir comment l'actualisation des textes du passé peut aider les élèves à percevoir et à comprendre les enjeux du contemporain, à partir d'une proposition d'étude des Liaisons dangereuses. Nous distinguerons trois phases: la phase d'appropriation de l'œuvre ${ }^{11}$, la phase collective, avec les communautés interprétatives, et la phase d'écriture, avec un accent particulier sur les types de travaux concevables dans une perspective actualisante.

\subsection{La phase d'appropriation de l'œuvre}

13 Il faut distinguer dans cette phase d'appropriation le travail préparatoire d'entrée dans le texte, et le travail plus approfondi sur le roman.

\subsubsection{L'entrée dans le texte}

\subsubsection{Les questionnaires}

14 Les questionnaires, décrits dans les propositions d'Anne Vibert sur la lecture subjective $^{12}$, sont une bonne manière d'entrer dans le texte, à partir de questions destinées à encourager l'implication subjective plutôt que les traditionnelles questions «techniques». Des questions «ouvertes» sur les personnages appréciés ou au contraire détestés, sur l'éventuel féminisme de $\mathrm{M}^{\mathrm{me}}$ de Merteuil, sur la moralité ou l'immoralité perçue du texte, sur l'image que les élèves se font de l'auteur à partir de son roman, sur la pertinence des intrigues à notre époque, ou encore sur la présence ou l'absence du sentiment amoureux dans le récit, ont ainsi permis de comprendre combien les expériences de lecture des élèves pouvaient diverger, et ont pu servir de point d'appui à une réflexion collective en classe, permettant d'appréhender la profonde ambiguïté de l'œuvre (son « indécidabilité »), mais aussi son actualité.

\subsubsection{Le travail de modélisation spatiale}

À l'occasion de ce questionnaire, il a aussi été demandé aux élèves de classer les personnages selon un double axe, un axe horizontal du cœur et de la raison, un axe vertical du vice et de la vertu, après un travail préalable de définition de ces quatre termes clés à partir de recherches sur le site Lexilogos, qui agrège des dictionnaires contemporains (comme le Trésor de la langue française) et des dictionnaires historiques (comme les dictionnaires de l'Académie française). Les évolutions éventuelles des personnages lors du récit ont été visualisées par un système de flèches. 



difficilement appréhendables. Si un personnage vertueux comme $\mathrm{M}^{\text {me }}$ de Rosemonde a été facile à situer pour les élèves, le placement de personnages "problématiques ", comme Valmont ou Cécile, a suscité des débats. Valmont, Danceny et Cécile sont apparus comme les personnages les plus mobiles, et de nombreux élèves ont représenté un trajet de la marquise de la raison vers le cœur. En amenant les élèves à s'interroger sur leurs divergences de lecture et sur les causes de l'évolution des personnages, on a pu faire percevoir collectivement la complexité morale des Liaisons.

\subsubsection{Le travail d'identification des valeurs centrales des personnages}

S'interroger sur la question des valeurs suppose à la fois un travail d'interprétation ${ }^{13}$, d'actualisation ${ }^{14}$, mais aussi de contextualisation ${ }^{15}$. Ce travail sur les valeurs a permis aux élèves de mieux saisir l'originalité des personnages (le basculement du sentiment religieux à la religion du sentiment pour $\mathrm{M}^{\mathrm{me}}$ de Tourvel, ou l'absence apparente de valeurs pour Cécile, qu'on a pu relier avec les élèves à la question de l'éducation féminine, cruciale pour Laclos). Tous ces éléments ont été source de questionnements collectifs et ont contribué à l'élaboration d'interprétations personnelles et cohérentes du texte.

\subsubsection{La place des élèves faibles lecteurs dans les activités liées à la problématique}

Le roman de Laclos peut être une occasion intéressante pour impliquer des élèves faibles dans le nécessaire travail de démontage des rouages du texte. Des tâches essentielles peuvent leur être confiées, comme l'établissement de la chronologie des lettres, le repérage des effets d'accélération de ralentissement, de densification de l'action, le calcul du nombre de lettres pour chaque énonciateur, la circulation des lettres (transmises, contrefaites, détournées...).

\subsubsection{Le travail du texte}

19 Il est possible de commencer le travail du texte proprement dit en s'appuyant sur la première lettre de Cécile, avant que les élèves n'aient lu l'œuvre, en leur proposant une version tronquée de la lettre, et en demandant aux élèves d'imaginer l'identité de la 
mystérieuse personne à genoux devant la jeune fille. La révélation par la suite qu'il ne s'agit que d'un cordonnier sera l'occasion d'établir des données sur Cécile (ignorance, naïveté...), mais aussi de réfléchir sur le statut "opaque» des lettres dans l'œuvre. Toujours à partir de cette première lettre, on peut s'appuyer sur les fautes syntaxiques du personnage pour opérer un travail de réécriture, ou bien faire réécrire la scène $d u$ point de vue du cordonnier...

L'oralisation des textes, dont Anne Vibert a souligné l'importance, est particulièrement judicieuse dans le cas des Liaisons. La maladresse et la naïveté de l'expression de Cécile peuvent aussi se traduire par l'élaboration d'un langage corporel adéquat, la mise en voix s'accompagnant alors d'un travail d'incarnation du personnage. Une activité comparable peut être accomplie pour les personnages de Valmont, de $\mathrm{M}^{\text {me }}$ de Merteuil ou de $\mathrm{M}^{\mathrm{me}}$ de Tourvel, à partir de l'annotation préalable du texte, de la rédaction de didascalies, ou de la recherche d'une gestuelle particulière (pourraient ainsi apparaître la dureté, la rigidité de la marquise, par opposition à la souplesse du vicomte, ou encore l'importance de l'expressivité du corps dans la rhétorique du sentiment de la présidente).

21 Il est également possible de consacrer une séance introductive à un travail sur les contradictions entre la préface et l'avertissement, en s'appuyant sur une élaboration collective du schéma argumentatif des deux énoncés. Dans une perspective plus spécifiquement actualisante, un débat oral peut être organisé à partir de la lettre 81, en demandant aux élèves s'ils pensent qu'une telle lettre aurait un sens aujourd'hui (ils seraient ainsi amenés à prendre conscience des progrès considérables de la condition féminine, mais aussi peut-être des nouvelles formes de contraintes et de conditionnements produits par notre époque). Tout ce travail pourra mener vers l'organisation de communautés interprétatives.

\subsection{Les communautés interprétatives et la question des valeurs}

La mise en place de communautés interprétatives - notion développée par Yves Citton à partir des théories de Stanley Fish ${ }^{16}$ - peut prendre trois formes dans le cadre de la classe, formes décrites par Anne Vibert à partir notamment de dispositifs exploités par Céline Billouard ${ }^{17}$ : le cercle de lecture, le débat interprétatif et le débat évaluatif. Ces dispositifs auront deux objectifs dans le cadre de l'étude des Liaisons dangereuses: un objectif méthodologique (favoriser l'entrée collective dans le texte, le faire exister dans l'espace de la classe, permettre une assimilation dynamique des enjeux), et un objectif "éthique ", de manière à utiliser le texte pour apporter un éclairage nouveau et original sur des problématiques contemporaines.

\subsubsection{Les cercles de lecture}

L'objectif de ces cercles ${ }^{18}$ est d'offrir un espace collectif de confrontation entre les lectures des élèves, soit dans le groupe classe, soit dans des groupes thématiques plus restreints, pour amener à une compréhension et à une perception des enjeux de l'œuvre. Les modalités de mise en place de cercles de lecture sont souples; elles peuvent être appuyées sur des éléments du carnet de lecture et également être "déterritorialisées", en utilisant par exemple le cadre du CDI. Le premier cercle de lecture portant sur Les Liaisons dangereuses pourrait être consacré à la détermination des objectifs et des obstacles de chaque personnage. Ce simple questionnement 
amènera les élèves à percevoir les multiples conflits, en repérant la modification des objectifs de la plupart des protagonistes, ce qui permettra de clarifier les intrigues $\left(\mathrm{M}^{\mathrm{me}}\right.$ de Merteuil devrait apparaître comme un obstacle, à la fois pour Cécile, pour Valmont et pour Danceny), ou de réfléchir aux contraintes (nécessité de la dissimulation, poids du regard du public et des rumeurs). Un autre cercle de lecture pourrait porter sur la situation de la femme dans le roman, ce qui pourrait ouvrir vers une réflexion " actualisée » sur la condition de la femme à notre époque. La question des origines et des effets de méchanceté de $\mathrm{M}^{\mathrm{me}}$ de Merteuil, à partir de la lettre 81, pourrait par ailleurs amener à une réflexion sur l'aliénation du féminin, sur le plaisir de nuire, sur la volonté de domination. Le dernier cercle pourrait concerner le dénouement. D'autres travaux sont possibles dans ce cadre: à partir du carnet de lecture, chaque élève pourrait choisir la lettre qui l'a le plus marqué, et expliquer pourquoi. Les impressions et les arguments notés dans le carnet pourraient être l'occasion d'échanges et de débats.

\subsubsection{Les débats interprétatifs}

Avec un texte aussi opaque et ambigu que les Liaisons, les possibilités de débats interprétatifs sont multiples: $\mathrm{M}^{\mathrm{me}}$ de Merteuil, monstre ou rebelle? Dénouement logique ou plaqué ? Valmont amoureux? Une œuvre féministe? Le couple des Liaisons pourrait-il exister aujourd'hui ? Dans ce cadre, il est possible que des oppositions entre les réactions des garçons et des filles conduisent à une réflexion sur le statut du féminin dans le cadre scolaire, et plus généralement dans nos sociétés contemporaines.

\subsubsection{Le débat évaluatif}

Un débat évaluatif, portant sur la valeur, l'intérêt, la pertinence de l'œuvre, pourra aussi être organisé, l'enjeu étant de dépasser les simples impressions et opinions lapidaires, pour aller vers le développement d'un argumentaire, et l'amorce d'un débat. Pour être efficaces, tous ces échanges oraux devront s'appuyer sur des travaux préparatoires écrits, et déboucher également sur des réinvestissements à l'écrit, tout en restant adossés à des retours réguliers sur l'œuvre.

\subsection{Le couple Lecture / Écriture}

26 Nous reprendrons les trois approches décrites par François Le Goff ${ }^{19}$ (" écrire dans », " écrire à côté ", " écrire sur ») pour présenter quelques suggestions, qui permettront de travailler l'écriture d'invention.

\subsection{1. Écrire dans}

27 François Le Goff regroupe les travaux consistant à greffer des écrits des élèves sur le texte étudié en s'efforçant d'en respecter les codes sous l'appellation "écrire dans ». À partir d'un travail de lecture croisée sur les portraits de $\mathrm{M}^{\text {me }}$ de Tourvel par Valmont et $\mathrm{M}^{\text {me }}$ de Merteuil dans les lettres 5 et 6 , il est ainsi possible de faire rédiger le portrait de Valmont par $\mathrm{M}^{\mathrm{me}}$ de Tourvel qui écrirait à $\mathrm{M}^{\mathrm{me}}$ de Rosemonde, puis par $\mathrm{M}^{\mathrm{me}}$ de Volanges avec la même destinataire (les deux postures argumentatives de la présidente et de la mère de Cécile - défense et accusation, pourraient entraîner des effets intéressants). La forme épistolaire du roman permet d'imaginer des lettres 
intercalées, écrites par des correspondants, ou par les protagonistes non épistoliers, comme M. de Tourvel. Faire écrire une lettre de prison par Prévan, destinée à se défendre et à faire accuser $\mathrm{M}^{\mathrm{me}}$ de Merteuil, permettrait à la fois de revenir sur la subtilité du piège construit par la marquise, et de développer un travail argumentatif.

\subsection{2. Écrire à côté}

On peut imaginer des sujets d'écritures actualisants qui consisteraient à "écrire à côté » de l'œuvre de Laclos, en restant dans le champ fictionnel, mais sans respecter le cadre générique, spatial ou temporel du texte source. À partir de la lettre 81, il serait ainsi possible d'amener les élèves à réfléchir au système de contraintes modernes dont sont victimes les filles dans notre société actuelle, en partant d'un travail de réécriture et de modernisation de la lettre. Toujours dans cette perspective actualisante, on peut concevoir un sujet qui amènerait les élèves à imaginer comment les deux libertins exploiteraient les réseaux sociaux, Facebook ou Twitter. Sur Facebook, quels types de messages posteraient-ils sur le Mur, lesquels seraient privés? Quels personnages accepteraient d'être leurs amis (grâce à sa duplicité, $\mathrm{M}^{\mathrm{me}}$ de Merteuil devrait avoir une liste sensiblement plus longue que le dangereux Valmont)? Ainsi que le suggère Maria Léone, il est également envisageable d'inciter les élèves à construire des pages Facebook pour chacun des personnages (notons cependant que de telles pages existent déjà). Un travail de transposition générique est par ailleurs possible, en partant d'une lettre fortement "dramatisée ", comme la lettre 85 , qu'il s'agirait de réécrire sous forme théâtrale pour la mettre en scène et la jouer.

\subsection{3. Écrire sur}

"Écrire sur " implique une approche métatextuelle, comme dans l'exercice du commentaire. Une réflexion sur la notion de vertu dans les Liaisons dangereuses pourrait être un sujet d'écriture très ambitieux et complexe. Le concept est opaque pour les élèves, et nécessitera un travail de recherche en amont (recherche lexicale, éventuels groupements de textes). Il s'agira ensuite de repérer le passage dans le roman d'une vertu de type chrétien (exprimée par la religiosité de $\mathrm{M}^{\mathrm{me}}$ de Tourvel, à une vertu de type rousseauiste, qui repose avant tout sur une authenticité du sentiment). Une fois la notion de vertu remise dans son contexte historique, il est possible de réfléchir sur le sens de la vertu dans nos sociétés contemporaines, sur les modalités particulières que ce concept pourrait prendre, soit par un questionnement dans le carnet de lecture, soit à l'occasion d'un cercle de lecture, soit dans un travail écrit. Par ailleurs, dans le cadre du cercle de lecture, certains élèves pourraient être chargés de faire un compte rendu des débats, à la manière de journalistes, ou travailler à la rédaction d'une synthèse.

Disons-le en guide de conclusion. Dans cet article, nous avons insisté sur la fonction de l'actualisation: briser la frontière invisible qui sépare l'œuvre patrimoniale de l'expérience vécue du lecteur, pour redonner aux œuvres du passé une pertinence qui permettra un travail dynamique du texte. Évidemment, les pistes évoquées ne sont que des ébauches, et les questions sont vastes et multiples, notamment celui de la redéfinition du corpus d'œuvres étudiées au regard de l'approche subjective et actualisante. En définitive, l'objectif de ces réflexions sera de modifier les déprimants résultats de l'enquête de Jean-François Massol, qui indiquait que le XvIII ${ }^{\mathrm{e}}$ siècle était le 
plus mal aimé des élèves, puisque les œuvres de ce siècle ne représentaient que 0,9\% des lectures déclarées aimées par les élèves ${ }^{20}$.

\section{NOTES}

1. Pour une description précise de l'approche actualisante, voir Y.Citton, Lire, interpréter, actualiser : pourquoi les études littéraires ?, Paris, éditions Amsterdam, 2007.

2. Certaines de ces propositions, en particulier celles concernant la phase d'appropriation de l'œuvre, ont été exploitées dans le cadre d'une classe de $1^{\text {re }} \mathrm{S}$ du lycée Europole de Grenoble, d'autres ne constituent que des pistes pédagogiques à explorer.

3. On peut songer au Salammbô de Druillet, ou à L'île au Trésor dessinée par Hugo Pratt. De nombreuses adaptations d'œuvres patrimoniales sont disponibles: des pièces de théâtre avec reprise intégrale des répliques, comme Les Précieuses ridicules ou Tartuffe, des adaptations de romans comme Madame Bovary, Le Père Goriot, À la recherche du temps perdu...

4. La notion de dystopie, centrale pour comprendre 1984 ou Le Meilleur des mondes, sera plus facile à appréhender pour l'élève si l'enseignant s'appuie sur des œuvres comme Hunger Games.

5. Au sujet des adaptations de Laclos, voir en particulier les actes du colloque Laclos après Laclos, éd. C. Seth, Paris, Hermann, 2016.

6. Hollywood maitrise très bien ce biais d'actualisation. On peut citer plusieurs adaptations modernisantes de Shakespeare : le Romeo et Juliette (1996) de Baz Luhrman, mais aussi 10 Things I Hate about You (1999) inspiré de La Mégère apprivoisée de Shakespeare, ou la version de Beaucoup de bruit pour rien (2012) réalisée par Joss Wheldon. Dans le domaine des séries télévisées, la version contemporaine de Sherlock créée par Steve Moffat pour la BBC est également un modèle d'actualisation.

7. Comme le montrent certaines illustrations de manuels présentées par Isabelle Roque dans son article " Le Molière actualisé des manuels de collège et de lycée », le risque est réel que les images produites apparaissent rapidement comme « datées ».

8. Pour une étude de ces anachronismes, voir Y. Citton, « Du bon usage de l'anachronisme (MarieAntoinette, Sofia Coppola et Gang of Four) », dans M. Poirson et L. Schifano (dir.), L'Écran des Lumières: regards cinématographiques sur le XVIII siècle, Oxford, Voltaire Foundation, 2009, p. 231-247.

9. Nombreuses sont les œuvres appartenant à l'horizon culturel des élèves qui permettent une actualisation de nature à éclairer les problématiques de textes du passé. Les comics et les films de super héros permettent de comprendre bien des enjeux de l'épopée (mythes fondant une communauté, notion de merveilleux, d'hyperbole...) ; la série des Twilight pour comprendre la sublimation du désir par sa frustration dans le cadre de l'amour courtois; quant à la série des Paranormal Activity, elle permet de revenir sur les éléments constitutifs du fantastique, de même que la série télévisuelle de Netflix, Stranger Things (2016)...

10. Dans un cadre différent de celui des réseaux sociaux, la nécessité du scandale et de la transgression, afin d'exister dans l'espace mondain, théorisée par Versac dans Les Égarements du cour et de l'esprit (1736-1738) de Crébillon, peut également être rapprochée des comportements des célébrités contemporaines obsédées par l'occupation de l'espace médiatique. Sur l'émergence du concept de célébrité au XVIII ${ }^{\mathrm{e}}$ siècle, voir A. Lilti, Figures publiques. L'invention de la célébrité (1750-1850), Paris, Fayard, coll. « L'épreuve de l'histoire », 2014. 
11. Ce sont essentiellement les propositions liées à cette phase d'appropriation qui ont été expérimentées en classe.

12. La plupart des stratégies ici décrites sont inspirées des dispositifs présentés par A. Vibert dans «Faire place au sujet lecteur en classe: quelles voies pour renouveler les approches de la lecture analytique au collège et au lycée ?», disponible sur <http://eduscol.education.fr>.

13. Certaines des questions qui ont été explorées dans le cadre de la séquence : l'amour est-il une valeur pour Valmont? $\mathrm{M}^{\mathrm{me}}$ de Merteuil a-t-elle vraiment aimé Valmont, comme elle le proclame ? La marquise a-t-elle des valeurs ? Le sens de l'honneur de Danceny est-il si profond? Y a-t-il une subversion des valeurs aristocratiques? Y a-t-il des liens forts entre sentiment et morale, comme semble vouloir le prouver Laclos?

14. C'est ainsi que l'exaltation des sentiments (le personnage de $M^{\text {me }}$ de Tourvel) est apparue comme actualisable, alors que le code aristocratique de l'honneur est apparu comme obsolète.

15. La contextualisation pourra notamment s'appuyer sur le travail de recherche dans les dictionnaires de l'époque et dans l'Encyclopédie, à partir du site Lexilogos.

16. Y. Citton, "Puissance des communautés interprétatives ", préface à S. Fish, Quand lire, c'est faire, Paris, éditions des Prairies ordinaires, 2007, p. 5-27.

17. C. Billouard, Réhabiliter la subjectivité de l'élève: le "moi» au cœur de la démarche interprétative, mémoire professionnel, IUFM de Grenoble - Université Joseph Fourier, année universitaire 2007-2008.

18. Sur les cercles de lecture, voir le travail des concepteurs du dispositif : S. Terwagne, S. Van Hulle et A. Lafontaine, Les cercles de lecture, Bruxelles, De Boeck, 2002.

19. F. Le Goff, «Les malles du lecteur, ou la lecture en écrivant », dans C. Mazauric, M.J. Fourtanier et G. Langlade (dir.), Textes de lecteurs en formation, Bruxelles, PIE Peter Lang, 2011, p. 219-229.

20. J.-F. Massol, «Spontanément ou par obligation, quand les lycéens lisent des œuvres », dans P. Demougin et J.-F. Massol (coord.), Lecture privée et lecture scolaire : la question de la littérature à l'école, CRDP de l'académie de Grenoble, 1999, p. 121.

\section{RÉSUMÉS}

Cet article se propose de réfléchir aux possibilités d'approches actualisantes d'un texte patrimonial de la littérature française - Les Liaisons dangereuses (1782) de Laclos - dans le cadre d'une classe de lycée. Après un travail de mise au point conceptuelle, pour décrire les diverses modalités d'actualisation possibles, une série de dispositifs sont proposés pour actualiser le roman en s'appuyant sur les expériences subjectives des élèves.

This communication intends to examine different "actualisation" strategies in the classroom, based on the study of a "patrimonial text" written by Laclos: Dangerous liaisons (1782). First of all, a variety of concepts are used to describe the different ways an "actualisation" process can be implemented in the classroom. Several "actualisation" strategies of laclos' novel are then proposed, in order to bring it closer to the students' subjective expérience 


\section{AUTEUR}

\section{DOMINIQUE HÖLZLE}

Université Grenoble Alpes, UMR 5316 LITT\&ARTS CNRS.

Dominique Hölze, agrégé de lettres modernes, docteur en littérature du XVIII ${ }^{\mathrm{e}}$ siècle, est chercheur associé de la composante CHARNIERES de l'UMR 5316 LITT\&ARTS CNRS de l'Université Grenoble Alpes. Il a coédité les Lettres athéniennes de Crébillon (Paris, 2010) et publié Le roman libertin au dix-huitième siècle : une esthétique de la séduction (Oxford, 2012). Il enseigne actuellement en lycée, à la Cité scolaire internationale Europole, à Grenoble. 